\title{
Appearance, fate and utilization of abnormal porcine embryos produced by in vitro maturation and fertilization
}

\author{
K. Kikuchi', T. Somfai'", M. Nakai' and T. Nagai ${ }^{2}$ \\ 'Division of Animal Sciences, National Institute of Agrobiological Sciences, Tsukuba, Ibaraki \\ 305-8602, Japan; ${ }^{2}$ National Institute of Livestock and Crassland Science, Tsukuba, Ibaraki, 305-0901, \\ Japan; "Present address: National Institute of Livestock and Crassland Science, Tsukuba, Ibaraki, \\ 305-0901, Japan
}

In vitro production (IVP) including in vitro maturation (IVM) and fertilization (IVF) is now an important technology for obtaining live piglets. However, there are still two significant obstacles to the efficient production of viable porcine embryos: (1) polyspermy and (2) fertilization of oocytes arrested at the immature stage. These phenomena relate to production of embryos with abnormal ploidy (polyploidy). To avoid these problems, careful selection of mature oocytes for IVF, and regular monitoring of normal and abnormal fertilization (polyspermy and/or lack of male pronucleus formation) are very important. In our recent studies, however, we have confirmed that some oocytes with abnormal ploidy after polyspermy can develop into diploid embryos with potentially normal developmental ability. The mechanism by which such fertilized polyploid oocytes develop to a normal state during embryo development is still not well understood. Attempts to clarify this mechanism would hopefully reveal data that are very useful for not only IVP but also other technologies such as the production of transgenic or cloned animals using IVM oocytes, including other species, also for human reproductive manipulation. In this review, we focus on studies of normality of IVM oocytes and ploidy of IVP embryos, and try to suggest practical ways of solving the problems mentioned above in pigs.

\section{Normality of porcine zygotes produced in vitro}

The in vitro developmental competence or viability of porcine in vitro-matured (IVM)-in vitrofertilized (IVF) oocytes to the blastocyst stage was first confirmed and reported by Mattioli et al. (1989). Since then, live-born piglets have been obtained from IVM-IVF embryos after in vitro culture (IVC) to the 2- to 4-cell stages (Mattioli et al. 1989; Yoshida et al. 1993; Funahashi et al. 1996; Funahashi \& Day 1997). Viable piglets have also been generated by transfer of in vitro-produced (IVP $=$ IVM, IVF and IVC) embryos at the blastocyst stage (Marchal et al. 2001; Kikuchi et al. 2002). Over the last few years, IVC procedures have been improved, but IVM and IVF systems are still hampered by problems resulting in poor developmental ability, and low quantity and quality of the embryos produced. This leads to embryo loss even after their transfer to recipients. One of the major causes of this problem is abnormal ploidy of IVP embryos due to (1) polyspermy during IVF and (2) fertilization of oocytes arrested at the 
immature stage. In addition, aneuploidy during oocyte maturation should be also noticed as a cause of embryo abnormality (Lechniak et al. 2007). It is essential to make every effort to achieve normality in IVP embryos after IVM and IVF. However, it is basically impossible to prevent precocious meiotic arrest or polyspermy in all cultured oocytes even using currently established technologies. Another approach for obtaining a good result (live offspring) under these conditions is to select fertilized oocytes or embryos that guarantee the developmental competence to piglets. It is generally accepted that only monospermic fertilized oocytes that have matured meiotically to the metaphase-II (M-II) stage can be guaranteed to show normal embryonic development. On the other hand, in comparison with sperm, oocyte sources are limited, especially those from rare genetic resources. To apply in vitro reproductive technologies in such animals, it is important to utilize as many oocytes as possible from a limited number of females. For this propose, assuming some ability to develop to term, abnormally generated embryos may be used to generate offspring. For half a century, mammalian embryos with abnormal ploidy have been known to be capable of surviving even into the post-implantation period (Piko \& Bomsel-Helmreich 1960; Bomsel-Helmreich 1971; Han et al. 1999a). However, the details of the mechanisms involved have remained unclear.

In the present review article focusing on porcine IVP systems, we summarize the status and consequences of generating abnormal embryos obtained as a result of polyspermy and also by fertilization before completion of meiotic maturation. We also consider the use of abnormally generated embryos to improve porcine IVP efficacy.

\section{Polyspermy}

Status of efforts to reduce polyspermy

Polyspermy occurs as a result of simultaneous penetration of an oocyte by two or more spermatozoa. The problem of polyspermy in IVP porcine systems is significant, and has remained unsolved for many years. The main reason for polyspermic fertilization seems to be the presence of a large number of spermatozoa at the site of IVF in the absence of a regulatory effect of the female reproductive tract to control the quantity and quality of spermatozoa. An optimal sperm concentration and time interval for IVF are basic requirements for avoiding an extremely high incidence of polyspermy (Nagai et al. 2006). In our laboratory, we have chosen batches of frozen-thawed epididymal sperm showing good penetration ability to conduct IVF by co-incubation of $1 \times 10^{5} \mathrm{sperm} / \mathrm{ml}$ with oocytes for $3 \mathrm{~h}$. Under these conditions, we are able to achieve reproducible sperm penetration beginning at $2 \mathrm{~h}$ post-insemination, the penetration rate reaching a plateau of around $80 \%$ at $4 \mathrm{~h}$ with a $60 \%$ polyspermy rate and an average of 3 spermatozoa per oocyte (Kikuchi et al. 2002, 2006). This means that only $20 \%$ of the oocytes subjected to IVF are monospermic. Another reason for polyspermy is considered to be the insufficient ability of IVM oocytes to block polyspermy due to a delayed or incomplete zona reaction and the imperfect characteristics of the zona caused by lack of exposure to oviduct fluid (Funahashi 2003).

Up to now, many attempts to decrease polyspermy have been reported. There have been several attempts to imitate in vivo conditions during IVF in order to regulate the number of spermatozoa near the oocytes. Some procedures have been based on the concept of allowing a small number of functional or capacitated spermatozoa to approach the oocytes, and these include the climbing-over-a-wall (COW) method (Funahashi \& Nagai 2000), straw IVF (Li et al. 2003) and the biomimetic microchannel IVF system (Clark et al. 2005). Other methods have aimed at improving the characteristics of oocytes (more specifically the zona pellucida: ZP), such as treatment of oocytes with oviductal glycoproteins before IVF (Kouba et al. 2000; McCauley 
et al. 2003a), and/or modulation of sperm capacitation, acrosome status or sperm binding to the ZP by techniques such as exposure of spermatozoa and oocytes to oviductal cells (Nagai \& Moor 1990), oviductal fluid (Kim et al. 1997), oviduct-specific glycoproteins (Kouba et al. 2000; McCauley et al. 2003a), follicular fluid (Funahashi \& Day 1993) or hyaluronan (Suzuki et al. 2000) before or during IVF. Although these approaches have been able to moderate the frequency of polyspermy, they could not eliminate it completely. Furthermore, some of these methods require special equipment (e.g. the biomimetic microchannel) or involve undefined factors (e.g. co-culture systems), and thus cannot be applied routinely to porcine IVP systems. As a consequence, in currently used IVF systems, the incidence of polyspermic fertilization is still very high. Thus, polyspermy is still considered to be a major problem that reduces the productivity of IVP systems in the pig.

\section{Selection of monospermic oocytes among IVF oocytes}

The most reliable method for selecting monospermic oocytes is to observe a single penetrated sperm head or male pronucleus in the ooplasm directly. Observation of a pronucleus(ei) is quite reliable, because oocytes with two (male and female) pronuclei are normally the result of monospermy, whereas oocytes with three or more pronuclei are the result of polyspermy. Unfortunately, the ooplasm in large animals such as pigs and cattle contains a large number of lipid droplets that make direct observation of organelles difficult. Pronuclei in such oocytes can be visualized by either nuclear staining or centrifugation. Vital nuclear staining is usually performed using fluorochromes such as Hoechest 33342, but this has been proven to have a detrimental effect on embryo development (Ebert et al. 1985; Tsunoda et al. 1988; Smith 1993; Yang et al. 1990).

Centrifugation is an effective way to polarize lipid droplets and thus to visualize pronuclei (Cran 1987), and seems to be safer than nuclear staining with fluorescent dye. To study the effects of centrifugation on IVP zygotes, we have evaluated the survival and developmental ability of fertilized oocytes after centrifugation (Somfai et al. 2008). In pigs, pronucleus formation is evident from $5 \mathrm{~h}$ post-insemination and reaches a plateau after $8 \mathrm{~h}$ (Kikuchi et al. 2006). Oocytes emitting 2 polar bodies, and thus considered to be mature and activated by sperm penetration, were centrifuged $10 \mathrm{~h}$ after insemination at $10,000 \times \mathrm{g}$ for $20 \mathrm{~min}$. This method rendered pronuclei visible even under a stereo microscope, and also allowed the oocytes to remain viable. They were then cultured in vitro for 6 days. The cleavage rates on Day 2 (Day 0 $=$ the day of IVF), and the rates of viable blastocysts and average numbers of cells within them on Day 6 did not differ significantly between the centrifugation and control groups. These results indicate that centrifugation itself does not affect developmental ability. After centrifugation, oocytes with 1, 2 or more pronuclei and unfertilized oocytes were separable under a stereo microscope by direct observation of pronuclei (Fig. 1). The efficacy of selection was verified by fixation and staining, and we found that, in most (75.0-82.8\%) of the selected oocytes, the number of pronuclei assessed by direct observation matched that assessed by nuclear staining (Somfai et al. 2008). We therefore considered that the selection of mono- and polyspermic oocytes by centrifugation followed by direct nuclear observation at the pronucleus stage was acceptable for further experiments.

\section{Development after polyspermic fertilization}

When zygotes with different numbers of pronuclei were selected and cultured separately, we found that the cleavage rates of oocytes with one or two pronuclei (considered to be 

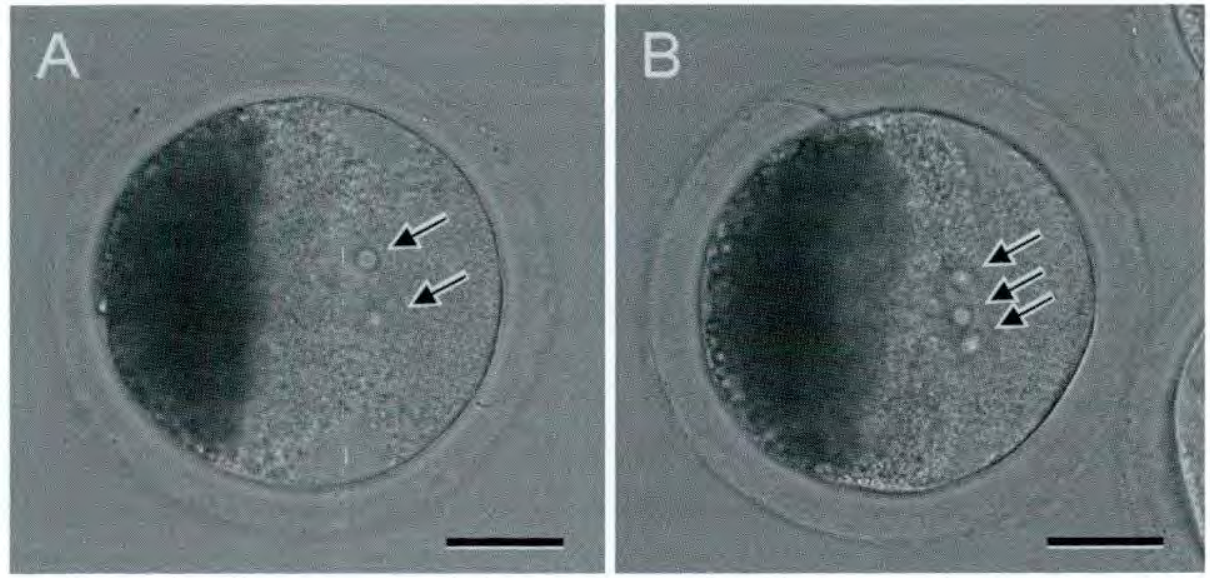

Fig. 1 Porcine oocytes after centrifugation treatment $10 \mathrm{~h}$ after IVF. Location and number of pronuclei are determined by the presence of nucleolus precursor bodies (arrows). A) Zygote with two pronuclei. B) Zygote with three pronuclei. Scale bar represents $30 \mu \mathrm{m}$.

A

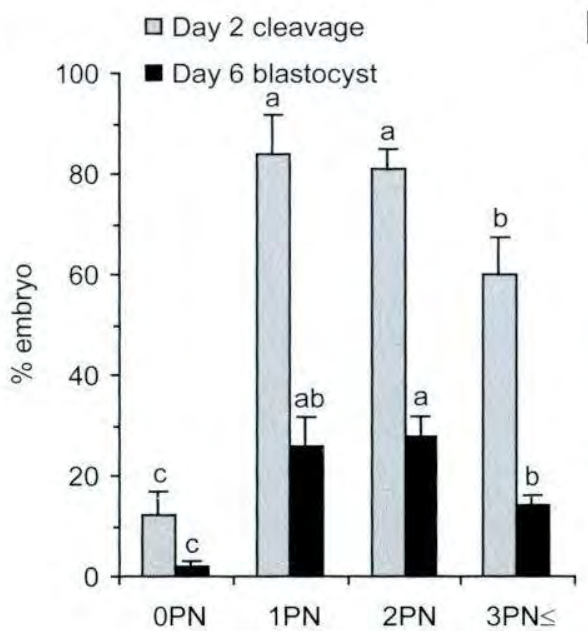

B

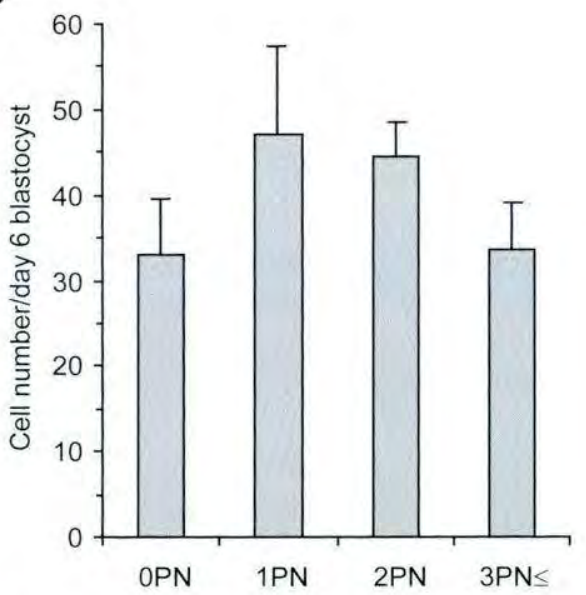

Pronucleus status after centrifugation

Fig. 2 In vitro development of in vitro-produced porcine zygotes with different numbers of pronuclei visualized by centrifugation. (A) Rates of cleaved embryos on Day 2 and blastocysts on Day 6. (B) Average cell number of blastocysts on Day 6. OPN; oocytes without pronucleus, $1 \mathrm{PN}$; oocyte with one pronucleus, 2PN; oocytes with two pronuclei, and $3 \mathrm{PN}<$; oocytes with three or more pronuclei. Mean \pm SEM are presented. ${ }^{\mathrm{a}-c}$ Percentages with different superscripts differ in each category $(\mathrm{P}<0.05)$. Data is from Somfai et al. (2008).

parthenogenetic or monospermic, respectively) were significantly higher than those of oocytes with three or more pronuclei (considered to be polyspermic) (Fig. 2) (Somfai et al. 2008). The rate of development to the blastocyst stage was higher for zygotes with two pronuclei (monospermy) than for those without any pronucleus or with three or more pronuclei (unfertilized oocytes 
or polyspermy, respectively), although it was not significantly different from that of zygotes with one pronucleus (parthenotes). The numbers of cells did not differ significantly among the oocytes thus classified. We analyzed the ploidy of blastocysts that had developed from putative monospermic and polyspermic zygotes (Table 1), and found that $73.8 \%$ of blastocysts that developed from monospermic oocytes were diploid ( $2 n$ ) whereas $12.5 \%$ of them were mixoploid containing a diploid cell(s). Thus, after calculation, $86.3 \%$ of the blastocysts that had developed from monospermic oocytes were found to contain diploid cells. On the other hand, $31.3 \%$ and $14.5 \%$ of polyspermic oocytes were found to develop into diploid blastocysts and mixoploid blastocysts containing a diploid cell(s), respectively. Calculation revealed that $45.8 \%$ of the blastocysts from polyspermic oocytes contained a diploid cell(s). These results suggest that not only monospermic oocytes but also a proportion of polyspermic oocytes may have the ability to develop to diploid embryos or fetuses.

Table 1. Ploidy of in vitro-cultured porcine blastocysts developing from zygotes bearing two or multiple pronuclei.

\begin{tabular}{|c|c|c|c|c|c|c|c|c|}
\hline \multirow[b]{2}{*}{$\begin{array}{l}\text { Pronuclear } \\
\text { status after } \\
\text { centrifugation }\end{array}$} & \multicolumn{7}{|c|}{$\%$ ploidy of blastocyst } & \multirow{2}{*}{$\begin{array}{c}\% \\
\text { blastocyst } \\
\text { with } 2 n \\
\text { cells }\end{array}$} \\
\hline & $\mathrm{n}$ & $2 n$ & $3 n$ & $4 n$ & $4 n<$ & $\begin{array}{l}\text { Mixoploid } \\
\text { with } 2 n \\
\text { cells }\end{array}$ & $\begin{array}{c}\text { Mixoploid } \\
\text { without } 2 \mathrm{n} \\
\text { cells }\end{array}$ & \\
\hline $2 \mathrm{PN}$ & $8.9 \pm 4.4$ & $73.8 \pm 11.9^{3}$ & 0 & $4.7 \pm 4.7$ & 0 & $12.5 \pm 12.5$ & 0 & $86.3 \pm 0.5^{a}$ \\
\hline $3 \mathrm{PN} \leq$ & $17.0 \pm 11.9$ & $31.3 \pm 10.1^{\mathrm{b}}$ & $12.1 \pm 6.5$ & $6.0 \pm 3.2$ & $3.7 \pm 3.7$ & $14.5 \pm 7.2$ & $15.1 \pm 2.6$ & $45.8 \pm 9.4^{b}$ \\
\hline
\end{tabular}

PN; pronuclei.

Mean \pm SEM are presented.

$a, b$ Percentages with different superscripts differ in each column $(P<0.05)$.

Table is modified from Somfai et al. (2008).

Our data agree with previous studies that found high frequencies of chromosomal abnormalities (mainly polyploidy) in IVP porcine embryos, which were thought to have resulted from polyspermic fertilization (McCauley et al. 2003b; Somfai et al. 2005). Polyspermic porcine embryos reportedly have an ability to develop to the blastocyst stage in vitro (Han et al. 1999a). This suggests that a considerable proportion of IVP blastocysts in those studies resulted from polyspermic fertilization. It has also been reported that, when polyspermic zygotes are transferred into recipients, most of the fetuses on Day 40 are diploid. This indicates that, in many polyspermic embryos, the effect of abnormally high numbers of penetrating spermatozoa on embryo ploidy is neutralized during in vivo development (Han et al. 1999b).

\section{Possible mechanism of ploidy correction in polyspermic embryos}

The mechanisms involved in the correction of polyploidy to a normal diploid status in polyspermic oocytes are still unclear. The ploidy of porcine embryos resulting from polyspermic fertilization is thought to depend on the location of the pronuclei before the first cleavage (Han et al. 1999b). This phenomenon is believed to be related to the different cleavage patterns of polyspermic embryos (including abnormal tripolar and tetrapolar cleavage), which can result in diploid, polyploid and also mixoploid embryos with a wide variety of blastomere ploidy after the first cell cleavage, including polyploids, diploids, and aneuploids (Han et al. 1999b; Funahashi 2003). It is believed that, during early development of embryos, blastomeres with abnormal ploidy fail to develop to the fetal stage whereas those with diploid blastomeres are able to do so. Thus, mosaic embryos having diploid cells may have the ability to develop to term (Han et al. 1999b, Funahashi et al. 2003). Other authors have suggested the involvement 
of a different mechanism; Kola et al. (1987) found that tripolar cleavage of polyspermic human oocytes always resulted in abnormal numbers of chromosomes, whereas the appearance of a protrusion in 2 cell embryos was associated with diploid status in polyspermic oocytes. Similar results were obtained recently in in vitro-produced bovine embryos (Somfai et al. 2009). Funahashi (2003) has mentioned another possible mode of ploidy correction, when the chromosome compartment (a male pronucleus or a decondensed sperm head) from one sperm does not contribute to syngamy and the formation of the mitotic metaphase. Such a male nucleus may remain in the cytoplasm of one of the blastomeres of the resulting 2-cell embryo, and later this extra chromatin is disrupted and disappears through a mechanism that involves lysosomes. In current IVP systems, a reasonable proportion (15-30\%) of porcine embryos can develop to the blastocyst stage, but with a wide variety of morphological appearances including partially fragmented embryos characterized by both nuclear and anuclear blastomeres (Kikuchi et al. 2002; Somfai et al. 2005; Somfai et al. 2008). Our recent study (Somfai et al. 2008) has also revealed that at $36 \mathrm{~h}$ after IVF a higher rate of cleaved monospermic than polyspermic embryos developed to the two-cell stage, whereas the proportion of embryos containing four or more blastomeres was significantly higher in the polyspermic than in the monospermic group. Nuclear staining revealed that the frequency of embryos containing bi- or multi-nuclear blastomere(s) did not differ between the monospermic and polyspermic groups, whereas the proportion of embryos containing at least one blastomere without any nucleus (considered as a sign of a fragmented cytoplasm) was significantly higher in polyspermic than in monospermic embryos. The mean number of blastomeres was significantly higher in polyspermic than in monospermic embryos, whereas the mean number of nuclei per embryo did not differ between the two groups (Table 2). These results suggest that polyspermic fertilization is associated with partial embryo fragmentation; however, it is not clear if this phenomenon is related to ploidy correction during early embryonic development.

Table 2. Ploidy of in vitro-cultured porcine blastocysts developing from zygotes bearing two or multiple pronuclei.

\begin{tabular}{|c|c|c|c|c|c|c|c|c|}
\hline \multirow[b]{2}{*}{$\begin{array}{l}\text { Pronuclear } \\
\text { status after } \\
\text { centrifugation }\end{array}$} & \multirow[b]{2}{*}{$\%$ cleaved } & \multicolumn{3}{|c|}{$\begin{array}{c}\%^{\mathrm{b}} \text { embryos by microscopic } \\
\text { evaluation }\end{array}$} & \multicolumn{4}{|c|}{ Nuclear staining } \\
\hline & & 2 cell & 3 cell & 4 cell $\leq$ & $\begin{array}{c}{ }^{\mathrm{b}} \text { embryos } \\
\text { with } \\
\text { multiple } \\
\text { nuclei }\end{array}$ & $\begin{array}{c}\text { \% b embryos } \\
\text { with } \\
\text { anuclear } \\
\text { blastomere }\end{array}$ & $\begin{array}{l}\text { Mean no. } \\
\text { blastomeres } \\
\text { per embryo }\end{array}$ & $\begin{array}{l}\text { Mean no. } \\
\text { nuclei } \\
\text { per } \\
\text { embryo } \\
\end{array}$ \\
\hline $2 \mathrm{PN}$ & $77.7 \pm 6.0^{c}$ & $56.0 \pm 6.0^{c}$ & $22.0 \pm 6.1$ & $24.9 \pm 7.0^{c}$ & $6.9 \pm 3.4$ & $34.4 \pm 3.1^{c}$ & $2.8 \pm 0.1^{c}$ & $2.3 \pm 0.1$ \\
\hline $3 \mathrm{PN} \leq$ & $59.7 \pm 6.4^{\mathrm{d}}$ & $26.7 \pm 4.1^{d}$ & $25.8 \pm 3.9$ & $47.7 \pm 6.6^{\mathrm{d}}$ & $13.9 \pm 1.3$ & $57.6 \pm 3.8^{\mathrm{d}}$ & $3.4 \pm 0.1^{\mathrm{d}}$ & $2.5 \pm 0.1$ \\
\hline
\end{tabular}

PN; pronucleus(ei).

All embryos were evaluated or fixed at $36 \mathrm{~h}$ after onset of IVF

a Percentage to the examined embryos

b Percentage to the cleaved embryos

Mean \pm SEM are presented.

a,bPercentages with different superscripts differ in each column $(P<0.05)$.

Table is modified from Somfai et al. (2008).

\section{Fertilization before completion of meiotic maturation}

\section{Cytoplasmic maturity of "metaphase-l-arrested" oocytes}

In our previous IVM system (Kikuchi et al. 1999a), matured oocytes at the M-II stage appeared at $30 \mathrm{~h}$ and the maturation rate became maximal after $36 \mathrm{~h}$ of IVM. About $35 \%$ of the cultured oocytes failed to reach the M-II stage and about $25 \%$ of them were arrested while still immature 
(Fig. 3). These oocytes were characterized by a typical metaphase plate without a first polar body. This phenomenon is often referred to as "metaphase-I (M-I) arrest". In general, meiotic arrest at the $\mathrm{M}-\mathrm{I}$ stage can be caused by a number of factors, such as insufficient meiotic competence because of an inadequate follicular phase or oocyte diameter (Szybek 1972; Sorensen \& Wassarman 1976; Motlik \& Fulka 1986; Eppig et al. 1994) or stress caused by inappropriate culture conditions, such as the use of inadequate isolation or culture media (Bae \& Foote 1980; Bagger et al. 1987; Kikuchi et al. 1999b). Intracellular calcium regulation reported to affect this phenomenon (Lechniak et al. 2005). As well as in pigs, in certain mouse strains (LT/Sv and LT-related strains), it is known that oocytes undergo meiotic arrest during the M-I stage (Hirao \& Eppig 1997; 1999).

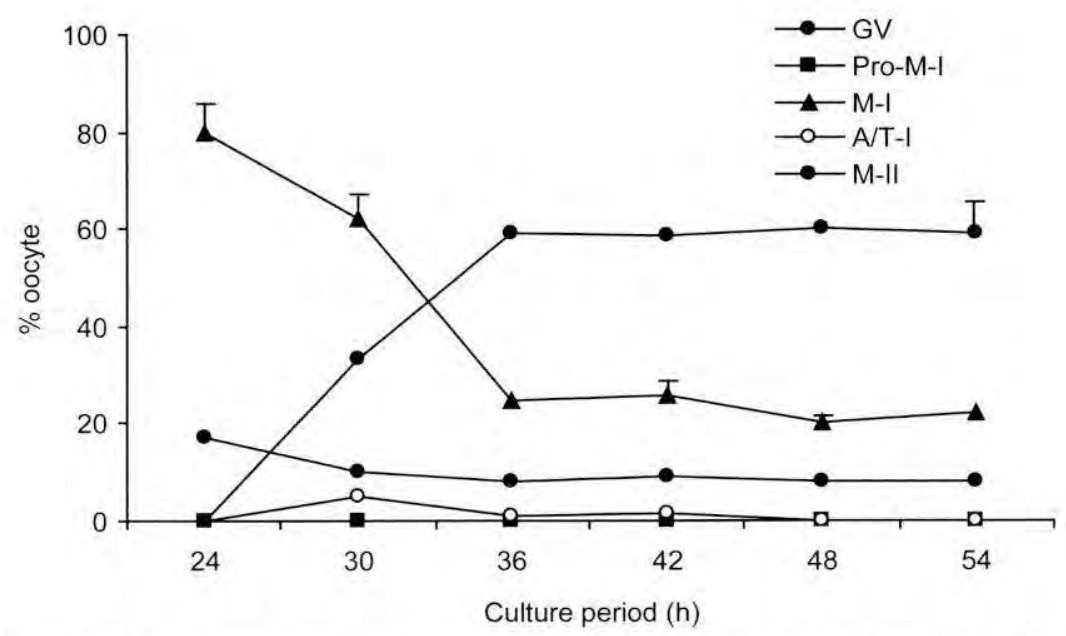

Fig. 3 Nuclear transition during in vitro maturation of porcine follicular oocytes. Oocytes were cultured and their nuclear status examined every $6 \mathrm{~h}$ from 24 to $54 \mathrm{~h}$. Data are presented as means \pm SE. GV, germinal vesicle; Pro-M-I, pro-metaphase-I; M-I, metaphase-I; A/T-I, anaphase-I or telophase-I; M-II, metaphase-II. The figure is modified from Kikuchi et al. (1999a).

Mature (M-II) oocytes can be distinguished from immature ones by direct observation of a first polar body with minimum error [less than $6 \%$ in the polar body (PB) (+) group, Kikuchi et al. 1999b]. Comparing with selection after staining with a fluorescent dye, this method is very easy to perform and does not seem to affect developmental ability (Ebert et al. 1985; Tsunoda et al. 1988; Smith 1993; Yang et al. 1990). Our previous study (Kikuchi et al. 1999b) showed that "M-l-arrested" oocytes completed their cytoplasmic maturation just like M-II oocytes, and were capable of activation to form male and female pronuclei after IVF and also the female pronucleus after parthenogenetic stimulation. Mature oocytes having a first polar body emitted a second polar body after oocyte activation, whereas "M-l-arrested" oocytes without a polar body were activated with emission of a single polar body (Table 3). The pronucleus status (timing of its formation and morphological features) did not differ between activated "M-I-arrested" and M-Il oocytes (Somfai et al. 2005). These results suggest that cytoplasmic maturation for oocyte activation can be completed after $48 \mathrm{~h}$, even when nuclear maturation has not completed the $\mathrm{M}$-II stage. On the other hand, maturing oocytes with insufficient cytoplasmic maturity [PB(-) oocytes after culture for $24 \mathrm{~h}$, considered to be "fresh" M-I] did not respond to parthenogenetic stimulation and only a few of them formed pronuclei after IVF. This difference was explained by a high level of maturation-promoting factor in "fresh" $\mathrm{M}-\mathrm{I}$ oocytes and a reduced level of 
this factor in oocytes that were cultured for $48 \mathrm{~h}$, regardless of the existence of a polar body (Kikuchi et al. 1999b). In fact, sperm heads that had penetrated into the immature cytoplasm of "fresh" M-I oocytes did not undergo transformation to a male pronucleus, but recondensed or transformed to metaphase-like chromosomes (Fig. 4). This also confirms the insufficient cytoplasmic ability of $\mathrm{M}-\mathrm{I}$ oocytes at an earlier maturational stage. These results suggest that, unlike maturing oocytes, oocytes arrested at an immature stage after germinal vesicle breakdown undergo cytoplasmic maturation that allows oocyte activation and male pronucleus formation after IVF, similar to the case of matured (M-II) oocytes.

Table 3. Activation of porcine oocytes classified on the basis of the presence of a polar body.

\begin{tabular}{|c|c|c|c|c|c|}
\hline \multirow{2}{*}{ Culture [h] } & \multirow{2}{*}{ Polar body* } & \multirow{2}{*}{ Treatment } & \multirow{2}{*}{$\%$ oocytes } & \multicolumn{2}{|c|}{$\%$ activated oocytes } \\
\hline & & & & With one polar body & With two polar bodies \\
\hline 24 & - & IVF & $6.2 \pm 1.5^{b}$ & $80.0 \pm 16.7^{a}$ & $20.0 \pm 16.7^{b}$ \\
\hline 48 & - & IVF & $68.6 \pm 10.8^{\mathrm{a}}$ & $90.0 \pm 1.7^{a}$ & $10.0 \pm 1.7^{b}$ \\
\hline 48 & + & IVF & $61.8 \pm 13.8^{\mathrm{a}}$ & $5.2 \pm 2.3^{b}$ & $94.9 \pm 2.3^{\mathrm{a}}$ \\
\hline 24 & - & ES & $0^{\mathrm{b}}$ & - & - \\
\hline 48 & - & ES & $52.9 \pm 5.0^{\mathrm{a}}$ & $73.0 \pm 9.7^{\mathrm{a}}$ & $13.5 \pm 4.7^{a}$ \\
\hline 48 & + & ES & $80.5 \pm 4.8^{\mathrm{a}}$ & $8.9 \pm 2.6^{b}$ & $79.0 \pm 6.7^{b}$ \\
\hline
\end{tabular}

*Examined by Nomarski differential interference contrast microscopy before treatment.

IVF; in vitro fertilization, ES; electrical stimulation

$a, b$ Percentages with different superscripts differ in each treatment (IVF or ES) $(P<0.05)$

Table is modified from Kikuchi et al. (1999b).
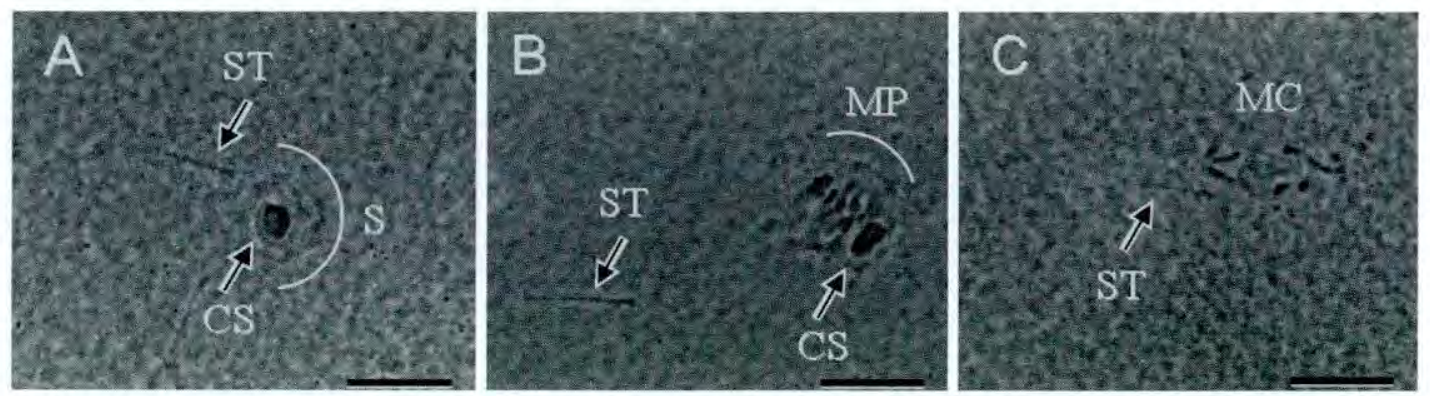

Fig. 4 Sperm heads penetrating an immature porcine oocyte at the metaphase-I stage. Oocytes were cultured for $24 \mathrm{~h}$, fertilized in vitro and subsequently cultured for up to 36 h. Arrows indicate the tails of the penetrated spermatozoa. After initial decondensation, sperm heads were recondensed into a mass (Cs), being surrounded by a spindle (S) or incorporated into the maternal metaphase plate $(\mathrm{Mp})(\mathrm{A}$ and $\mathrm{B}$, respectively), or transformed into metaphase-like chromosomes (C). Scare bars represent $10 \mu \mathrm{m}$. The figure is modified from Kikuchi et al. (1999b).

\section{Developmental competence of "M-I-arrested" oocytes}

"M-I-arrested" porcine oocytes seem to have some developmental ability, as they were shown to develop to the blastocyst stage after IVF, similarly to M-II oocytes (Somfai et al. 2005). In mice also, "M-I-arrested" oocytes have been shown to be capable of developing to the blastocyst stage (Eppig et al. 1994). The developmental competence of meiotically arrested porcine oocytes is rather poor, resulting in low rates of formation of blastocysts, which contain low numbers of cells (Table 4). Chromosomal analysis of blastocysts resulting from IVF of "M-I- 
arrested" porcine oocytes has revealed a high frequency of chromosome numerical abnormality in blastomeres, especially an increased rate of triploidy (Table 5). Similar results have already been reported in mice (Eppig et al. 1994). Fertilization of "M-l-arrested" oocytes is suggested to result in the development of digynic triploid embryos. As reported by Kaufman et al. (1989), triploid diandric mouse embryos appear morphologically normal, but are smaller than normally fertilized diploid embryos.

Table 4. Blastocyst formation after IVF of porcine oocytes with/without a polar body after culture for $44 \mathrm{~h}$.

\begin{tabular}{cccccc}
\hline \multirow{2}{*}{ Polar body* } & \% blastocysts & \multicolumn{2}{c}{$\%$ blastocysts categorized morphologically as } & \multicolumn{2}{c}{$\begin{array}{c}\text { Cell number per } \\
\text { blastocyst }\end{array}$} \\
\cline { 3 - 5 } & & Excellent & Good & Poor & $29.1 \pm 1.4^{\mathrm{b}}$ \\
\hline- & $20.7 \pm 2.8^{\mathrm{b}}$ & $60.0 \pm 5.1$ & $20.0 \pm 8.5^{\mathrm{b}}$ & $20.0 \pm 2.0^{\mathrm{a}}$ & $2.1 \pm .^{\mathrm{b}}$ \\
+ & $34.6 \pm 2.4^{\mathrm{a}}$ & $68.1 \pm 5.1$ & $23.0 \pm 3.8^{\mathrm{b}}$ & $8.7 \pm 1.4^{\mathrm{b}}$ & $52.0 \pm 2.5^{\mathrm{a}}$ \\
\hline
\end{tabular}

*Examined by stereo microscopy before IVF.

IVF; in vitro fertilization, ES; electrical stimulation

$a, b$ Percentages with different superscripts differ in each column $(P<0.05)$.

Table is modified from Somfai et al. (2005).

Table 5. Chromosome analysis of blastomeres in Day 6 blastocysts generated from porcine oocytes with/without a polar body.

\begin{tabular}{cccccccc}
\hline & \multirow{2}{*}{$\begin{array}{c}\text { No. of } \\
\text { Polar body } \\
\text { blastocysts } \\
\text { examined }\end{array}$} & $\begin{array}{c}\text { No. of } \\
\text { metaphases } \\
\text { prepared (per } \\
\text { blastocyst) }\end{array}$ & Total $^{\mathrm{d}}$ & Haploid $^{\mathrm{b}}$ & Diploid $^{\mathrm{b}}$ & Triploid $^{\mathrm{b}}$ & Tetraploid $^{\mathrm{b}}$ \\
\cline { 4 - 8 }+- & 66 & $132(2.0)$ & $100(78.8)^{c}$ & $16(16.0)$ & $44(44.0)^{\mathrm{d}}$ & $34(34.0)^{\mathrm{c}}$ & $6(6.0)$ \\
+ & 122 & $241(2.0)$ & $155(64.3)^{\mathrm{d}}$ & $29(18.7)$ & $108(69.7)^{\mathrm{c}}$ & $13(8.4)^{\mathrm{d}}$ & $5(3.2)$ \\
\hline
\end{tabular}

a Percentage relative to the number of prepared metaphases

bercentage relative to the number of analyzed metaphases

c,d Percentages with different superscripts differ in each column $(P<0.05)$

Table is modified from Somfai et al. (2005).

The ploidy of porcine embryos, as suggested previously, might be related to their developmental competence. The existence of a ploidy correction mechanism like that operating in polyspermy has not yet been confirmed in zygotes or embryos from "M-l-arrested" oocytes. Nevertheless, diploid cells have been found in blastocysts that have developed from "M-l-arrested" oocytes, suggesting that the embryos are diploid, or are mixoploid but contain diploid cells. Detailed chromosome analysis will be needed to clarify the nuclear competence of such embryos.

\section{Possible mechanism of "M-I arrest" before completion of oocyte maturation}

We have compared the chromosome configurations of "M-l-arrested" oocytes with those of $\mathrm{M}-\mathrm{I}$ oocytes at $33 \mathrm{~h}$ of IVM ("real M-I" oocytes) and with those of M-II oocytes at $48 \mathrm{~h}$ of IVM (Somfai et al. 2006) (Fig. 5). In a series of experiments, we matured porcine oocytes in the presence of the actin polymerization inhibitor cytochalasin-B (CB), a drug that inhibits actin filament polymerization. This drug has been reported to induce meiotic arrest of mammalian oocytes at the M-I stage (Wassarman et al. 1976). Constant tracking of nuclear progression during maturation revealed that segregation of homologous chromosomes did, in fact, occur in CB-treated oocytes; however, extrusion of the first polar body failed. This caused the formation of two sets of segregated homologous chromosomes inside the oocyte. Later, these sets united and formed a single, tetraploid metaphase plate (Somfai et al. 2006) (Fig. 6). Similar observations 

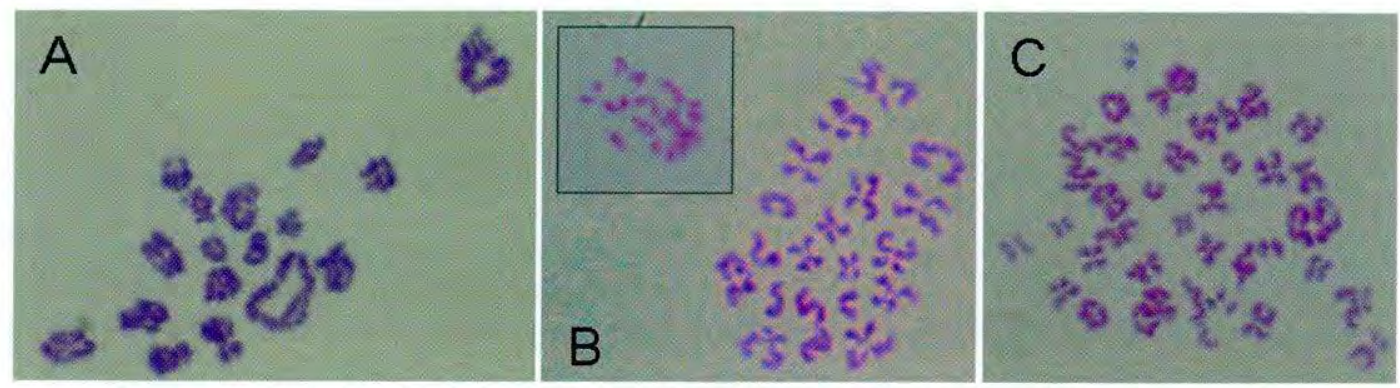

Fig. 5 Morphology of porcine oocyte chromosomes. (A) chromosomes prepared from oocytes at the metaphase-I (M-I) stage (33 h IVM), where 19 ring-shaped, symmetrical complexes of the homologous chromosomes from bivalents are observed; (B) chromosomes prepared form oocytes at the metaphase-II (M-II) stage (44 h IVM). Insert: chromosomes incorporated by a first polar body; and (C) chromosomes prepared from oocytes cultured in the presence of cytochalasin-B (44 h IVM). Both M-II- and CB-treated oocytes displayed similar separate chromosomes with two chromatids; M-II oocytes had 19 chromosomes, whereas the number of chromosomes in the CB-treated oocytes was double (38) compared with M-II oocytes. The figure is modified from Somfai et al. (2006).
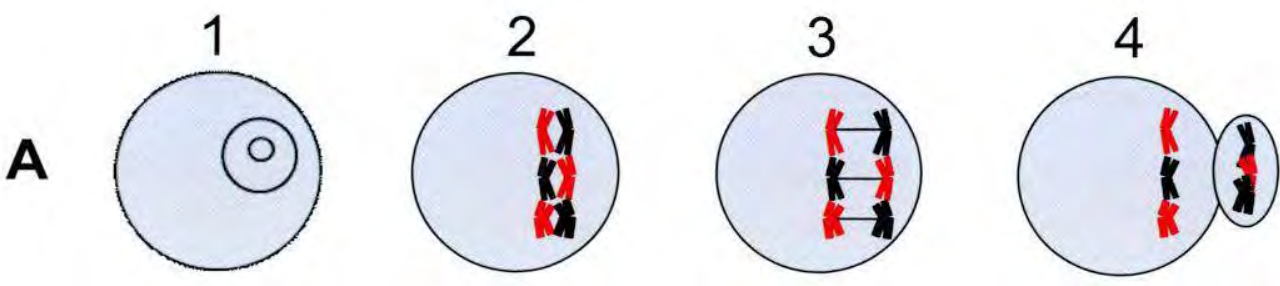

\section{6 h IVM (no CB)}

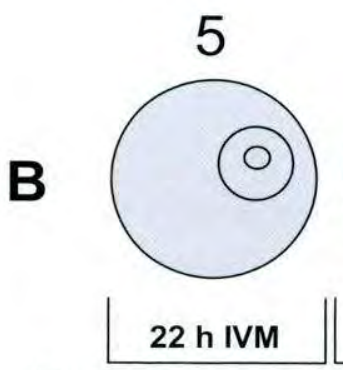

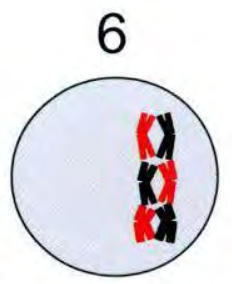

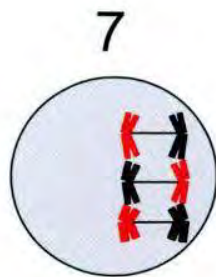

22 h IVM + CB
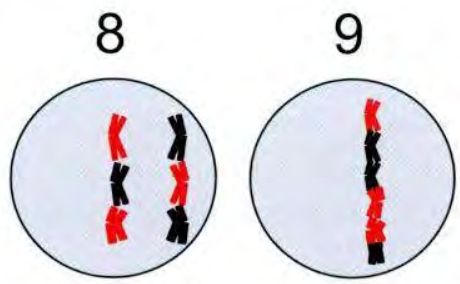

2 h IVM (no CB)

Fig. 6 Scheme of meiotic maturation without (A) or with (B) cytochalasin-B (CB). (A) Germinal vesicle (GV) oocytes (1) are cultured in vitro without $C B$ for 46 h. After the GVBD (around 33 under these culture conditions), the homologous paternal and maternal chromosomes form bivalents [metaphase-I (M-I) stage (2)]. At this stage, the oocyte is tetraploid. Later segregation of the homologous chromosomes occurs (3) with extrusion of the first polar body [metaphase-II (M-II) stage (4)], so the oocyte becomes diploid. (B) After culture of GV oocytes for $22 \mathrm{~h}$ in CB-free medium (5), they are subsequently matured in the presence of $\mathrm{CB}$ for $22 \mathrm{~h}$. The $\mathrm{M}-\mathrm{I}$ stage (6) is followed by segregation of homologous chromosomes (7), but extrusion of the polar body is inhibited, so that all chromosomes remain in the oocytes (8). During the last phase of maturation culture, the segregated chromosomes undergo rearrangement into an M-I-like metaphase plate (9). The figure is modified from Somfai et al. (2006). 
were published previously by Kubiak et al. (1991), who cultured mouse oocytes in the presence of cytochalasin-D, a drug similar to $C B$. The metaphase plate of porcine oocytes after treatment with CB contained 2n (38) segregated chromosomes, unlike those of "real M-I" oocytes that contained 19 pairs of homologous chromosomes forming bivalents and those of $\mathrm{M}$-II oocytes that contained $n$ (19) segregated chromosomes with 2 chromatids. Therefore the structure of the metaphase plates in "M-I-arrested" oocytes generated by CB treatment appears similar to that of M-II oocytes; however, it contains a double (diploid) set of 2 chromatids. Therefore such a nuclear stage cannot be considered a "real M-I" stage, but rather an "M-l-like" stage.

Previously, we compared the chromosome complements of CB-treated oocytes with those of "M-I-arrested" oocytes that were blocked spontaneously during IVM, and found exactly the same chromosome configurations (Fig. 7) (Somfai et al. unpublished results). This suggests that, during meiotic progression from the $\mathrm{M}-\mathrm{I}$ to $\mathrm{M}$-II stages, an as-yet unclarified mechanism gives rise to a similar actin-depolymerization-related phenomenon of chromosome reunion and rearrangement, even after successful segregation of the homologous chromosomes. The resulting nuclear stage is characterized by a single metaphase plate without any polar body, and has been referred to as "M-I arrest" in our previous papers (Kikuchi et al. 1999b; Somfai et al. 2005). This abnormality during porcine oocyte maturation occurs with increased frequency as culture is prolonged (Sosnowski et al. 2003). This suggests the malfunctions of the spindle during maturation culture. Further studies of these phenomena will be important not only in porcine IVP system, but also in human reproduction, because incompletion of oocyte maturation before the M-II stage is considered to be an important cause of human infertility (Mrazek \& Fulka 2003).
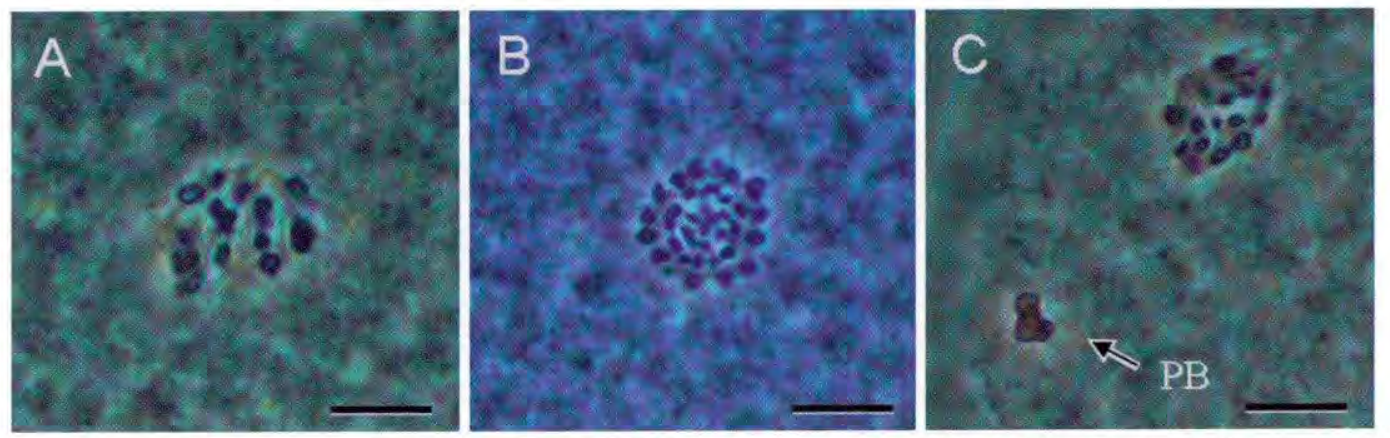

Fig. 7 Chromosome configurations of (A) metaphase-I (cultured for 33 h), (B) "metaphaseI-arrested" (for $48 \mathrm{~h}$ ) and (C) metaphase-II porcine oocytes. Specimens were prepared after fixation and staining with aceto-orcein, and examined by phase-contrast microscopy. Scale bars represent $10 \mu \mathrm{m}$.

\section{Conclusion}

Our results have clearly revealed that development to the blastocyst stage is not a perfect indicator of embryo quality in porcine IVP systems, since polyploid embryos can develop to the blastocyst stage. Careful selection of M-II oocytes for IVF, and regular monitoring of fertilization and polyspermy rates are very important in order to obtain reliable results, not only in porcine IVP systems but also in other technologies using IVM oocytes. On the other hand, it is necessary to know more about the mechanism by which fertilized oocytes with abnormal ploidy can develop to a normal state during embryo development. Further improvements of IVM-IVF systems are necessary to increase the degree of nuclear and cytoplasmic maturity and to reduce the incidence of polyploidy in porcine IVP systems. 


\section{Acknowledgments}

The authors would like to thank Drs. N. Kashiwazaki, H. Kaneko, J. Noguchi, M. Fahrudin, NWK Karja, M. Ozawa, N. Maedomari, for completion of a series of experiments, and also thank Ms. T. Aoki and Mr. Y. Funakubo for technical assistance

\section{References}

Bae IH \& Foote RH 1980 Maturation of rabbit follicular oocytes in a defined medium of varied osmolality. Journal of Reproduction and Fertility 5911-13.

Bagger PV, Byskov AG \& Christiansen MD 1987 Maturation of mouse oocytes in vitro is influenced by alkalization during their isolation. Journal of Reproduction and Fertility $\mathbf{8 0}$ 251-255.

Bomsel-Helmreich O 1971 Fate of heteroploid embryos. Advances in the Biosciences 6 381-403.

Clark SG, Haubert K, Beebe DJ, Ferguson CE \& Wheeler MB 2005 Reduction of polyspermic penetration using biomimetic microfluidic technology during in vitro fertilization. Lab on a Chip 5 1229-1232.

Cran DG 1987 The distribution of organelles in mammalian oocytes following centrifugation prior to injection of foreign DNA. Gamete Res 18 67-76.

Ebert KM, Hammer RE \& Papaioannou VE 1985 A simple method for counting nuclei in the preimplantation mouse embryo. Experimentia 41 1207-1209.

Eppig JJ, Schultz RM, O'Brien M \& Chesnel F 1994 Relationship between the developmental programs controlling nuclear and cytoplasmic maturation of mouse oocytes. Developmental Biology 164 1-9.

Funahashi $\mathbf{H} 2003$ Polyspermic penetration in porcine IVMIVF systems. Reproduction, Fertility \& Development 15 167-177.

Funahashi H, Kim NH, Stumpf TT, Cantley TC \& Day BN 1996 Presence of organic osmolytes in maturation medium enhances cytoplasmic maturation of porcine oocytes. Biology of Reproduction 54 1412-1419.

Funahashi H \& Day BN 1993 Effects of follicular fluid at fertilization in vitro on sperm penetration in pig oocytes. Journal of Reproduction and Fertility 99 97-103.

Funahashi H \& Day BN 1997 Advances in in vitro production of pig embryos. Journal of Reproduction and Fertility, Supplement 52 271-283.

Funahashi H \& Nagai, T 2000 Sperm selection by a climbing-over-a-wall IVF method reduces the incidence of polyspermic penetration of porcine oocytes. Journal of Reproduction and Development 46 319-324.

Han YM, Abeydeera LR, Kim JH, Moon HB, Cabot RA, Day BN \& Prather RS 1999a Growth retardation of inner cell mass cells in polyspermic porcine embryos produced in vitro. Biology of Reproduction $\mathbf{6 0}$ 1110-1113.

Han YM, Wang WH, Abeydeera LR, Petersen AL, Kim JH, Murphy C, Day BN \& Prather RS 1999b Pronuclear location before the first cell division determines ploidy of polyspermic pig embryos. Biology of Reproduction 61 1340-1346.

Hirao Y \& Eppig JJ 1997 Analysis of the mechanism(s) of metaphase I arrest in strain LT mouse oocytes: participation of MOS. Development $\mathbf{1 2 4}$ 5107-5113.

Hirao Y \& Eppig JJ 1999 Analysis of the mechanism(s) of metaphase l-arrest in strain LT mouse oocytes: delay in the acquisition of competence to undergo the metaphase I/anaphase transition. Molecular Reproduction and Development 54 311-318.

Kaufman MH, Lee KK \& Speirs S 1989 Influence of diandric and digynic triploid genotypes on early mouse embryogenesis. Development 105 137-145.

Kikuchi K, Naito K, Noguchi J, Shimada A,Kaneko H, Yamashita M, Tojo H \& Toyoda Y 1999a Inactivation of $\mathrm{p} 34^{\text {cdc } 2}$ kinase by the accumulation of its phosphorylated forms in porcine oocytes matured and aged in vitro. Zygote 7 173-179.

Kikuchi K, Nagai T, Ding J, Yamauchi N, Noguchi J \& Izaike $Y 1999$ b Cytoplasmic maturation for activation of pig follicular oocytes cultured and arrested at metaphase-I. Journal of Reproduction and Fertility 116 143-156.

Kikuchi K, Onishi A, Kashiwazaki N, Iwamoto M, Noguchi J, Kaneko H, Akita T \& Nagai T 2002 Successful piglet production after transfer of blastocysts produced by a modified in vitro system. Biology of Reproduction 66 1033-1041.

Kikuchi K, Nakai M, Shimada A \& Kashiwazaki N 2006 Production of viable porcine embryos by IVF and ICSI. Journal of Mammalian Ova Research 23 96-106.

Kim NH, Day BN, Lim JG, Lee HT \& Chung KS 1997 Effects of oviductal fluid and heparin on the fertility and characteristics of porcine spermatozoa. Zygote 5 61-65.

Kola I, Trounson A, Dawson G \& Rogers P 1987 Tripronuclear human oocytes: altered cleavage patterns and subsequent karyotypic analysis of embryos. Biology of Reproduction 37 395-401.

Kouba AJ, Abeydeera LR, Alvarez IM, Day BN \& Buhi WC 2000 Effects of porcine oviduct-specific glycoprotein on fertilization, polyspermy and embryonic development in vitro. Biology of Reproduction 63 242-250.

Kubiak J, Paldi A, Weber M \& Maro B 1991 Genetically identical parthenogenetic mouse embryos produced by inhibition of the first meiotic cleavage with cytochalasin D. Development 111 763-769.

Lechniak D, Szczepankiewicz D, Kauss D, Szulc J \& Szydłowski M 2005 IVM media, oocyte diameter 
and donor genotype at RYR1 locus in relation to the incidence of porcine diploid oocytes after maturation in vitro. Theriogenology 64 202-212

Lechniak D, Warzych E, Pers-Kamczyc E, Sosnowski J, Antosik P \& Rubes J 2007 Gilts and sows produce similar rate of diploid oocytes in vitro whereas the incidence of aneuploidy differs significantly. Theriogenology 68 755-762.

Li YH, Ma W, Li M, Hou Y, Jiao LH \& Wang WH 2003 Reduced polyspermic penetration in porcine oocytes inseminated in a new in vitro fertilization (IVF) system: straw IVF. Biology of Reproduction 69 1580-1585.

Marchal R, Feugang JM, Perreau C, Venturi E, Terqui M \& Miemillod P 2001 Meiotic and developmental competence of prepubertal and adult swine oocytes. Theriogenology 56 17-29.

Mattioli M, Bacci ML, Galeati G \& Seren E 1989 Developmental competence of pig oocytes matured and fertilized in vitro. Theriogenology 39 1201-1207.

McCauley TC, Buhi WC, Wu GM, Mao J, Caamano IN, Didion BA \& Day BN 2003a Oviduct-specific glycoprotein modulates sperm-zona binding and improves efficiency of porcine fertilization in vitro. Biology of Reproduction 69 828-834.

McCauley TC, Mazza MR, Didion BA, Mao J, Wu G, Coppola G, Coppola GF, Di Berardino D \& Day BN 2003b Chromosomal abnormalities in Day-6, in vitro-produced pig embryos. Theriogenology 60 1569-1580.

Motlik J \& Fulka J 1986 Factors affecting meiotic competence in pig oocytes. Theriogenology 25 87-96.

Mrazek M \& Fulka / 2003 Failure of oocyte maturation: possible mechanisms for oocyte maturation arrest. Human Reproduction 18 2249-2252.

Nagai T \& Moor RM 1990 Effect of oviduct cells on the incidence of polyspermy in pig eggs fertilized in vitro. Molecular Reproduction and Development 26 377-382.

Nagai T, Funahashi H, Yoshioka K \& Kikuchi K 2006 Update of in vitro production of porcine embryos. Frontiers in Bioscience 11 2565-2573.

Piko L \& Bomsel-Helmreich O 1960 Triploid rat embryos and other chromosomal deviants after colchicine treatment and polyspermy. Nature 186 737-739.

Smith LC 1993 Membrane and intracellular effects of ultraviolet irradiation with Hoechst 33342 on bovine secondary oocytes matured in vitro. lournal of Reproduction and Fertility 99 39-44.

Somfai T, Kikuchi K, Medvedev SU, Onishi A, Iwamoto M, Fuchimoto D, Ozawa M, Noguchi N, Kaneko H, Ohnuma K, Sato E \& Nagai T 2005 Development to the blastocyst stage of immature pig oocytes arrested before the metaphase-II stage and fertilized in vitro. Animal Reproduction Science 90 307-328.
Somfai T, Ozawa M, Noguchi ), Kaneko H, Ohnuma K, Karja NWK, Farhudin M, Maedomari N, Dinnyés A, Nagai T \& Kikuchi K 2006 Diploid porcine parthenotes produced by the inhibition of first polar body extrusion during in vitro maturation of follicular oocytes. Reproduction 132 559-570.

Somfai T, Ozawa M, Noguchi J, Kaneko H, Karja NWK, Fahrudin $M$, Nakai $M$, Maedomari N, Dinnyés A, Nagai T \& Kikuchi K 2008 In vitro development of polyspermic porcine oocytes: relationship between early fragmentation and excessive number of penetrating spermatozoa. Animal Reproduction Science 107 131-147.

Somfai $T$, Inaba $Y$, Aikawa $Y$, Ohtake $M$, Kobayashi S, Konishi K \& Imai K 2009 Relationship between the length of cell cycles, cleavage pattern, and developmental competence during in vitro culture of in vitro-matured/in vitro-fertilized bovine oocytes (Abstract). Reproduction, Fertility \& Development 21 209-210.

Sorensen RA \& Wassarman PM 1976 Relationship between growth and meiotic maturation of the mouse oocyte. Developmental Biology 50 531-536.

Sosnowski J, Waroczyk M \& Switonski 2003 Chromosome abnormalities in secondary pig oocytes matured in vitro. Theriogenology 60 571-581.

Suzuki H, Saito Y, Kagawa N \& Yang X 2003 In vitro fertilization and polyspermy in the pig: factors affecting fertilization rates and cytoskeletal reorganization of the oocytes. Microcopy Research and Technique 61 327-334.

Szybek K 1972 In vitro maturation of oocytes from sexually immature mice. Journal of Endocrinology 54 527-528.

Tsunoda Y, Shiosa Y, Onodera M, Nakamura K \& Uchida $T 1988$ Differential sensitivity of mouse pronuclei and zygote cytoplasm to Hoechst staining and ultraviolet irradiation. Journal of Reproduction and Fertility $\mathbf{8 2}$ 173-178.

Wassarman PM, Josefowicz WJ \& Letourneau GE 1976 Meiotic maturation of mouse oocytes in vitro: inhibition of maturation at specific stages of nuclear progression. Journal of Cell Science 12 531-545.

Yang X, Zhang L, Kovacs A, Tobback C \& Foote RH 1990 Potential of hypertonic medium treatment for embryo micromanipulation. II. Assessment of nuclear transplantation methodology, isolation, subzona insertion, and electrofusion of blastomeres to intact or functionally enucleated oocytes in rabbits. Molecular Reproduction and Development 27 118-129.

Yoshida M, Mizoguchi Y, Ishiaki K, Kojima T \& Nagai T 1993 Birth of piglets derived from in vitro fertilization of pig oocytes matured in vitro. Theriogenology 39 1303-1311. 